\title{
Rate-One Space-Time Block Codes With Full Diversity
}

\author{
Liang Xian and Huaping Liu, Member, IEEE
}

\begin{abstract}
Orthogonal space-time block codes provide full diversity, and maximum-likelihood (ML) decoding for orthogonal codes can be realized on a symbol-by-symbol basis. It has been shown that rate-one complex orthogonal codes do not exist for systems with more than two transmit antennas. For a general system with $N$ transmit and $M$ receive antennas, it is very desirable to design rate-one complex codes with full diversity. In this letter, we provide a systematic method of designing rate-one codes (real or complex) for a general multiple-input multiple-output system. Full diversity of these codes is then achieved by constellation rotation. A generalized, reduced-complexity decoding method for rate-one codes is also provided.
\end{abstract}

Index Terms-Diversity, space-time block codes (STBCs), wireless communications.

\section{INTRODUCTION}

$\mathbf{M}$ ULTIPATH fading could severely degrade the performance of wireless communication systems. As an effective method to combat the effects of fading, transmit diversity has been studied extensively in the past. The transmit diversity scheme proposed by Alamouti [1] is a simple and effective orthogonal space-time block code (STBC) of rate one for systems with two transmit antennas. Because of its simplicity in implementation and the feasibility of having multiple antennas at the base station, this scheme has been deployed in existing mobile communications systems. In [2], Tarokh et al. extended the orthogonal design to systems with an arbitrary number of transmit antennas and provided a class of rate-one real orthogonal codes. It was also proved that complex orthogonal design with transmission rate one does not exist for more than two transmit antennas (see [2, Th. 5.4.2] and [3] for nonsquare designs). In [4], Boariu et al. discussed a method to construct a class of rate-one nonorthogonal STBCs with partial diversity. Real Hadamard matrices played a key role in the scheme proposed in [4]. A class of quasi-orthogonal STBCs for systems with four transmit antennas was proposed in [5]-[7]. These quasi-orthogonal codes provide partial diversity but transmission rate one. To achieve full diversity for quasi-orthogonal codes for four transmit antennas, constellation-rotation schemes were proposed in [8] and [9]. Compared with the STBCs in [10]-[12], quasi-orthogonal codes result in constant power for each transmit antenna with constant amplitude modulation, which is desirable for practical implementation. Sethuraman et al. proposed a family of STBCs from division algebras [13], some of which are of full diversity, rate one, with a constant power. However, these rate-one codes

Paper approved by A. H. Banihashemi, the Editor for Coding and Communication Theory of the IEEE Communications Society. Manuscript received January 27, 2004; revised November 5, 2004 and May 15, 2005.

The authors are with the School of Electrical Engineering and Computer Science, Oregon State University, Corvallis, OR 97331 USA (e-mail: hliu@eecs.orst.edu).

Digital Object Identifier 10.1109/TCOMM.2005.860081 with a constant power have higher decoding complexities and worse performance than quasi-orthogonal codes with optimal constellation rotation.

In this letter, we provide a systematic method for designing rate-one codes, real or complex, with full diversity and minimum intersymbol interference (ISI) ${ }^{1}$ for a general multipleinput multiple-output (MIMO) system. Through this systematic method, existing quasi-orthogonal codes for four or less transmit antennas are extended to systems with a larger number of transmit antennas. The proposed code structures are based on the real orthogonal codes given in [2], and can be used for both real and complex signals. Although the codes derived are nonorthogonal for complex signals, the orthogonality property is used in the ISI minimization process. Because of ISI, the decoder for these nonorthogonal codes is more complex than that for orthogonal codes. Thus, we provide a generalized, reduced-complexity decoding algorithm for the proposed codes. This decoding method can also be applied for orthogonal codes.

\section{SYSTEM MODEL}

We consider a wireless communication system with $N$ transmit antennas in the base station and $M$ receive antennas in the remote. The transmission matrix is expressed as

$$
\boldsymbol{G}=\left[\begin{array}{cccc}
c_{1}^{1} & c_{1}^{2} & \ldots & c_{1}^{N} \\
c_{2}^{1} & c_{2}^{2} & \ldots & c_{2}^{N} \\
\vdots & \vdots & \ddots & \vdots \\
c_{L}^{1} & c_{L}^{2} & \ldots & c_{L}^{N}
\end{array}\right]
$$

where $c_{l}^{n}, l=1, \ldots, L, n=1, \ldots, N$, is the symbol transmitted from antenna $n$ at time $l$. Generally, elements of $G$ are linear combinations of input symbols $\left(s_{1}, s_{2}, \ldots, s_{K}\right)$ and their complex conjugates. For most widely used codes, however, each element of $\boldsymbol{G}$ is related to a single input symbol $s_{k}$ (e.g., in the form of $s_{k} e^{j \phi}, \phi \in[0,2 \pi)$ ). The codes given in [1], [2], [5]-[7], and [13] are all of this type, which is also our focus in this letter. Note that complex conjugate and sign change of $s_{k}$ are special cases of $s_{k} e^{j \phi}$. The code rate is defined as $R=K / L$. At time slot $l$, signals $\left\{c_{l}^{1}, \ldots, c_{l}^{N}\right\}$ are transmitted simultaneously from the $N$ transmit antennas, and the average power of data streams from each transmit antenna is normalized to $1 / N$.

We consider a frequency-nonselective Rayleigh fading channel. Thus, the path gain from transmit antenna $n$ to receive antenna $m$, denoted as $h_{n, m}$, is modeled as samples of independent complex zero-mean Gaussian random variables (RVs) with variance 0.5 per real dimension. The wireless channel is assumed to be quasi-static, so that the path gains are constant over a frame of length $L$ and change independently from one frame to another.

\footnotetext{
${ }^{1}$ Note that ISI here refers to the mutual interference among input symbols transmitted from different antennas and different time slots within a frame.
} 
Let the received signal by antenna $m$ at time $l$ be $r_{l}^{m}$. The receiver model is expressed by

$$
\boldsymbol{R}=\sqrt{\frac{\rho}{N}} \boldsymbol{G H}+\mathbf{W}
$$

where $\boldsymbol{R}=\left\{r_{l}^{m}\right\}_{1 \leq l \leq L, 1 \leq m \leq M}$ is the $L \times M$ received signal matrix, $\boldsymbol{H}=\left\{h_{n, m}\right\}_{1 \leq n \leq N, 1 \leq m \leq M}$ is the $N \times M$ channel fading coefficient matrix, $\mathbf{W}=\left\{w_{l}^{m}\right\}_{1 \leq l \leq L, 1 \leq m \leq M}$ is the $L \times M$ additive noise matrix whose elements are samples of independent zero-mean complex Gaussian RVs with variance $1 / 2$ per real dimension, and $\rho$ is the signal-to-noise ratio (SNR) per receive antenna.

With perfect channel state information, the maximum-likelihood (ML) receiver computes metric

$$
d=\left\|R-\sqrt{\frac{\rho}{N}} G H\right\|_{F}^{2}
$$

over all possible transmitted signal sets $\left(s_{1}, s_{2}, \ldots, s_{K}\right)$ and decides in favor of the set that minimizes $d\left(\|\cdot\|_{\mathrm{F}}\right.$ stands for Frobenius norm).

\section{Generalized Quasi-ORTHOgOnAl CODES}

\section{A. Code Construction}

Since our focus is on codes for which each element of $G$ is related to a single input symbol, it is clear from (3) that $\boldsymbol{G}^{H} \boldsymbol{G}$ $\left((\cdot)^{H}\right.$ stands for conjugate transpose $)$ is the only source of ISI. If all off-diagonal elements of $\boldsymbol{G}^{H} \boldsymbol{G}$ are zero, the transmission is ISI-free. Thus, the orthogonal design (columns of $G$ are mutually orthogonal) has zero ISI, and all transmitted symbols for such design can be separated by using a ML decoder.

To minimize ISI in rate-one design, we could maximize the number of zero off-diagonal elements, as all nonzero off-diagonal elements of $\boldsymbol{G}^{H} \boldsymbol{G}$ have the same statistics, and therefore contribute the same amount of ISI. Quasi-orthogonal codes are constructed based on maximizing the number of orthogonal column pairs in the code matrix. Quasi-orthogonality should hold for any complex input symbols, not just some specific complex symbols. Additionally, for real symbols, the code matrix should be an orthogonal matrix, so that it is suitable for both real and complex symbols. Thus, the proposed code has the same structure as the real orthogonal codes given in [2], but differs in that some elements are changed to their conjugates. Before detailing the systematic construction method for the type of codes described in the beginning of Section III-A, let us prove two propositions.

Proposition 1: Any two column vectors that are both orthogonal to the third column vector must be nonorthogonal to each other.

Proof: Recall that we only consider code matrices, each entry of which is related to a single input symbol. For rate-one transmission with constant power, a necessary condition to achieve full diversity without feedback is that each input symbol appears in each row and each column of the code matrix only once. Consider three column vectors $\boldsymbol{a}, \boldsymbol{b}$, and $\boldsymbol{c}$. If $\boldsymbol{a}$ is orthogonal to $\boldsymbol{b}$ and $\boldsymbol{a}$ contains any input symbol $s_{k}$ (could be any point in a complex plane), then $\boldsymbol{b}$ must contain $s_{k}^{*}$, where $(\cdot)^{*}$ stands for complex conjugate. For the same reason, $s_{k}^{*}$ must appear once in $\boldsymbol{c}$. Since both $\boldsymbol{b}$ and $\boldsymbol{c}$ contain $s_{k}^{*}$, they are nonorthogonal to each other. An indirect proof is that if Proposition 1 were not true, then one should find three mutually orthogonal columns. This implies that a rate-one complex orthogonal design for three transmit antennas exists, which leads to a conclusion that contradicts the theorem given in [2, Th. 5.4.2].

Proposition 2: The maximum number of orthogonal column pairs of a $K \times N$ code matrix is $(N / 2)^{2}$ when $N$ is even, and $\left(N^{2}-1\right) / 4$ when $N$ is odd.

Proof: With the restrictions of the design being considered, the code matrix has a size of $K \times N(K=L$ for rate-one design). Consider a complex rate-one code matrix. Without loss of generality, we assume that the first column is orthogonal to a maximum of $\alpha$ other columns. To maximize the total number of orthogonal column pairs, we could let each of the remaining $N-\alpha-1$ columns be orthogonal to all of the $\alpha$ columns. According to Proposition 1, the total number of orthogonal column pairs is $\alpha(N-\alpha)$. Let $f(\alpha)=\alpha(N-\alpha)$. It is easy to determine the maximum values of $f(\alpha)$ for integer $\alpha$ in the range $1 \leq \alpha \leq N-1$. These values are found to be $(N / 2)^{2}$ for even $N$, and $(((N-1) / 2) \times((N+1) / 2))$ for odd $N$, which can be realized by dividing all columns into two groups and making any column of one group orthogonal to all columns of the other group. The number of columns in the two groups must be kept $(N-1) / 2$ and $(N+1) / 2$ for odd $N$, and $N / 2$ for even $N$.

For complex codes, rate-one design can be generalized by fixing the positions and signs of the symbols in the real orthogonal design and then changing half (the top half, the bottom half, or the middle half) of all rows to their complex conjugates. Let us consider changing the top half as an example. For square rate-one real orthogonal codes when $K$ is an integer power of two, we can always partition $G$ into four subblocks as

$$
\boldsymbol{G}=\left[\begin{array}{cc}
\boldsymbol{G}_{-(1 \cdots K / 2)}^{a} & \boldsymbol{G}_{-(K / 2+1 \cdots K)}^{a} \\
\boldsymbol{G}_{-(K / 2+1 \cdots K)}^{b} & \boldsymbol{G}_{-(1 \cdots K / 2)}^{b}
\end{array}\right]
$$

for real design, and

$$
\boldsymbol{G}=\left[\begin{array}{cc}
\boldsymbol{G}_{-(1 \cdots K / 2)}^{a *} & \boldsymbol{G}_{-(K / 2+1 \cdots K)}^{a *} \\
\boldsymbol{G}_{-(K / 2+1 \cdots K)}^{b} & \boldsymbol{G}_{-(1 \cdots K / 2)}^{b}
\end{array}\right]
$$

for complex design. Consequently, any column in the left half of $G$ is orthogonal to any column in its right half. This method thus maximizes the number of orthogonal column pairs according to Proposition 2. Similarly, we can prove that changing the bottom half or the middle half of all rows also achieves the maximum number of orthogonal column pairs. Although different choices of these rows will affect the positions of the nonzero elements in $\boldsymbol{G}^{H} \boldsymbol{G}$, the total number of nonzero elements is the same. Therefore, these codes have the same performance.

We provide below a few examples of complex rate-one codes obtained by applying the proposed method. These codes are 
based on changing the middle rows of the real codes presented in [2] as

$$
\begin{aligned}
\boldsymbol{G}_{4} & =\left[\begin{array}{cccc}
s_{1} & s_{2} & s_{3} & s_{4} \\
-s_{2}^{*} & s_{1}^{*} & -s_{4}^{*} & s_{3}^{*} \\
-s_{3}^{*} & s_{4}^{*} & s_{1}^{*} & -s_{2}^{*} \\
-s_{4} & -s_{3} & s_{2} & s_{1}
\end{array}\right] \\
\boldsymbol{G}_{8} & =\left[\begin{array}{cccccccc}
s_{1} & s_{2} & s_{3} & s_{4} & s_{5} & s_{6} & s_{7} & s_{8} \\
-s_{2} & s_{1} & s_{4} & -s_{3} & s_{6} & -s_{5} & -s_{8} & s_{7} \\
-s_{3}^{*} & -s_{4}^{*} & s_{1}^{*} & s_{2}^{*} & s_{7}^{*} & s_{8}^{*} & -s_{5}^{*} & -s_{6}^{*} \\
-s_{4}^{*} & s_{3}^{*} & -s_{2}^{*} & s_{1}^{*} & s_{8}^{*} & -s_{7}^{*} & s_{6}^{*} & -s_{5}^{*} \\
-s_{5}^{*} & -s_{6}^{*} & -s_{7}^{*} & -s_{8}^{*} & s_{1}^{*} & s_{2}^{*} & s_{3}^{*} & s_{4}^{*} \\
-s_{6}^{*} & s_{5}^{*} & -s_{8}^{*} & s_{7}^{*} & -s_{2}^{*} & s_{1}^{*} & -s_{4}^{*} & s_{3}^{*} \\
-s_{7} & s_{8} & s_{5} & -s_{6} & -s_{3} & s_{4} & s_{1} & -s_{2} \\
-s_{8} & -s_{7} & s_{6} & s_{5} & -s_{4} & -s_{3} & s_{2} & s_{1}
\end{array}\right] .
\end{aligned}
$$

The Alamouti scheme can be considered as a special case of the proposed design. By changing the last row of the real orthogonal matrix

$$
\left[\begin{array}{cc}
s_{1} & s_{2} \\
-s_{2} & s_{1}
\end{array}\right]
$$

we obtain the complex design

$$
G_{2}=\left[\begin{array}{cc}
s_{1} & s_{2} \\
-s_{2}^{*} & s_{1}^{*}
\end{array}\right]
$$

which is the Alamouti scheme. Note that orthogonality still holds for this $2 \times 2$ complex code. The design $\boldsymbol{G}_{4}$ from the proposed method and the code matrix for four transmit antennas given in [5]

$$
\boldsymbol{A}_{4}=\left[\begin{array}{cccc}
s_{1} & s_{2} & s_{3} & s_{4} \\
-s_{2}^{*} & s_{1}^{*} & -s_{4}^{*} & s_{3}^{*} \\
-s_{3}^{*} & -s_{4}^{*} & s_{1}^{*} & s_{2}^{*} \\
s_{4} & -s_{3} & -s_{2} & s_{1}
\end{array}\right]
$$

have the same performance for complex signals as $\boldsymbol{G}_{4}^{H} \boldsymbol{G}_{4}$ and $\boldsymbol{A}_{4}^{H} \boldsymbol{A}_{4}$ have an equal number of nonzero elements, and these nonzero elements have identical statistical properties (mean and variance). However, the transmission matrix in [5] does not completely eliminate ISI for real input signals, whereas the proposed design does. Additionally, the proposed design can be applied to systems with an arbitrary number of transmit antennas, whereas the Jafarkhani scheme [5] is restricted to systems with four transmit antennas.

\section{B. Generalized Fast Decoding}

As it is well known, orthogonal codes can be decoded symbol by symbol. In other words, other symbols do not cause any interference in the process of decoding a particular symbol. Therefore, to decode any symbol, all other symbols can be assumed to be zero in the code matrix without performance loss. For example, to decode $s_{1}$ for the Alamouti scheme, one could let $s_{2}=0$ so that the code matrix becomes

$$
\left[\begin{array}{cc}
s_{1} & 0 \\
0 & s_{1}^{*}
\end{array}\right] \text {. }
$$

The decision variable for $s_{1}$ is obtained as $\hat{s}_{1}=\sum_{m=1}^{M}\left(h_{1, m}^{*} r_{1}^{m}+h_{2, m} r_{2}^{m *}\right)$, which is the same as the result derived in [1]. Applying this technique to various types of orthogonal codes (e.g., rate-1, 1/2, or 3/4 codes), one can easily find the decision variable without the lengthy calculation that is otherwise needed. Similarly, for quasi-orthogonal codes, such as $\boldsymbol{G}_{8}$, group $\left(s_{1}, s_{2}, s_{7}, s_{8}\right)$ can be decoded by letting $s_{3}=s_{4}=s_{5}=s_{6}=0$. For large-size constellations, complexity even with the fast ML decoding algorithm could still be very high. In this case, the sphere decoder (lattice decoder) [14] could be applied after the generalized fast ML decoder to further lower complexity.

\section{Optimal Constellation Rotation for Quasi-Orthogonal Codes}

Constellation rotation for $4 \times 4$ codes has been addressed in [8] and [9]. Thus, we only consider $\boldsymbol{G}_{8}$ in this letter. Consider $\boldsymbol{G}_{8_{-}(1,2,7,8)}$, which is formed by letting $s_{3}=s_{4}=s_{5}=s_{6}=0$ in $\boldsymbol{G}_{8}$. The minimum rank of $\boldsymbol{G}_{8_{-}(1,2,7,8)}(\mathcal{S})-\boldsymbol{G}_{8_{-}(1,2,7,8)}(\widetilde{\mathcal{S}})$ is four for some common modulation schemes, such as phase-shift keying (PSK) and quadrature amplitude modulation (QAM), where $\mathcal{S}=\left[s_{1}, s_{2}, s_{7}, s_{8}\right]^{T}$. Therefore, $\boldsymbol{G}_{8}$ only provides a diversity order four without constellation rotation. Full diversity can be achieved by constellation rotation only if the determinant of $\boldsymbol{G}_{8_{-}(1,2,7,8)}(\mathcal{S})-\boldsymbol{G}_{8_{-}(1,2,7,8)}(\widetilde{\mathcal{S}})$ is always nonzero.

For quasi-static flat Rayleigh fading channels, the upper bound of the pairwise error probability is given as [9], [15]

$$
P(\boldsymbol{G} \rightarrow \widetilde{\boldsymbol{G}}) \leq \frac{1}{2}\left(\prod_{i=1}^{r} \lambda_{i}\right)^{-M}\left(\frac{\rho}{4 N}\right)^{-r M}
$$

where $\boldsymbol{G}$ and $\widetilde{\boldsymbol{G}}$ are two different but arbitrarily chosen code matrices, $r=\min (\operatorname{rank}(\boldsymbol{G}-\widetilde{\boldsymbol{G}}))$ is the diversity order, and $\lambda_{1}, \lambda_{2}, \ldots, \lambda_{r}$ are the nonzero eigenvalues of $(\boldsymbol{G}-\widetilde{\boldsymbol{G}})(\boldsymbol{G}-$ $\widetilde{\boldsymbol{G}})^{H}$. The minimum value of the product $\lambda_{1}, \lambda_{2}, \ldots, \lambda_{r}$ over all pairs of distinct code matrices is defined as the coding gain. For $\boldsymbol{G}_{8}$ with full diversity, $\operatorname{det}[\boldsymbol{G}-\widetilde{\boldsymbol{G}}]=\left(\operatorname{det}\left[(\boldsymbol{G}-\widetilde{\boldsymbol{G}})^{H}\right]\right)^{*} \neq 0$. Maximizing the coding gain is equivalent to maximizing the minimum absolute value of the determinant of $(\boldsymbol{G}-\widetilde{\boldsymbol{G}})$.

Consider the group $\left(s_{1}, s_{2}, s_{7}, s_{8}\right)$ with

$$
\begin{aligned}
b= & \left|\operatorname{det}\left[\boldsymbol{G}_{8_{-}(1,2,7,8)}-\widetilde{G}_{8_{-}(1,2,7,8)}\right]\right| \\
= & \mid\left(s_{1}-\tilde{s}_{1}\right)^{2}+\left(s_{2}-\tilde{s}_{2}\right)^{2} \\
& +\left(s_{7}-\tilde{s}_{7}\right)^{2}+\left.\left(s_{8}-\tilde{s}_{8}\right)^{2}\right|^{4} .
\end{aligned}
$$

Let $s_{1}$ be the reference symbol with a rotation angle zero (without rotation), and rotation angles for $s_{2}, s_{7}$, and $s_{8}$ be $\theta_{1}$, $\theta_{2}$, and $\theta_{3}$, respectively. Equation (5) can then be rewritten as

$$
\begin{aligned}
b=\mid\left(s_{1}-\tilde{s}_{1}\right)^{2}+ & e^{j 2 \theta_{1}}\left(s_{2}-\tilde{s}_{2}\right)^{2} \\
& +e^{j 2 \theta_{2}}\left(s_{7}-\tilde{s}_{7}\right)^{2}+\left.e^{j 2 \theta_{3}}\left(s_{8}-\tilde{s}_{8}\right)^{2}\right|^{4} .
\end{aligned}
$$

It is very difficult to determine the optimal values of the three variables $\left(\theta_{1}, \theta_{2}, \theta_{3}\right)$ simultaneously via an analytical approach. Moreover, the optimal rotation angles are constellation-dependent. Through exhaustive numerical search and careful inspection, it is found that when the minimum value of $b$ is maximized, (6) can always be written as

$$
\begin{aligned}
b=\mid\left[\left(s_{1}-\tilde{s}_{1}\right)^{2}\right. & \left.+e^{j 2 \theta^{\prime}}\left(s_{2}-\tilde{s}_{2}\right)^{2}\right] \\
& +\left.e^{j 2 \theta}\left[\left(s_{7}-\tilde{s}_{7}\right)^{2}+e^{j 2 \theta^{\prime}}\left(s_{8}-\tilde{s}_{8}\right)^{2}\right]\right|^{4} .
\end{aligned}
$$

where $\theta^{\prime}=\pi / 4$ for QAM and $\theta^{\prime}=\pi / Q$ for PSK with a constellation size $Q$, when $Q$ is an integer power of two. Note that $\theta^{\prime}$ is 


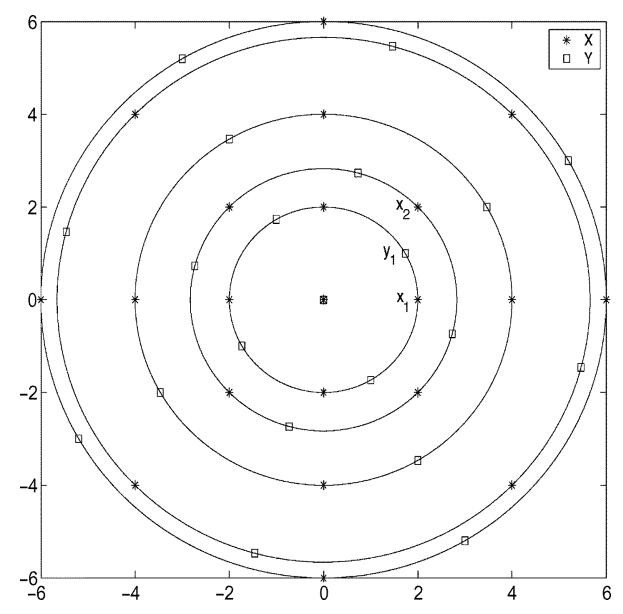

Fig. 1. Constellation $\mathcal{X}$ and $\mathcal{Y}=e^{j \pi / 6} \mathcal{X}$.

the optimal rotation angle for quasi-orthogonal codes with four transmit antennas [9], [16]. This leaves $\theta$ the only variable to be determined. In other words, if we let $\mathcal{X}$ be the new constellation formed by $\left(s_{1}-\tilde{s}_{1}\right)^{2}+e^{j 2 \theta^{\prime}}\left(s_{2}-\tilde{s}_{2}\right)^{2}$, then maximizing the minimum value of $b$ is equivalent to maximizing the minimum distance between any point in $\mathcal{X}$ and any point in $\mathcal{Y}=e^{j 2 \theta} \mathcal{X}$. If the minimum distance is nonzero (except between the origins of constellations $\mathcal{X}$ and $\mathcal{Y}$ ), then full diversity is achieved.

For 4QAM symbols $\{(\sqrt{2} / 2) \pm(\sqrt{2} / 2) j,-(\sqrt{2} / 2) \pm$ $(\sqrt{2} / 2) j\}$, the optimal value of $\theta$ and the corresponding $b$ can be determined by using the geometric method given in [16]. Fig. 1 shows five circles with radii $\{2,2 \sqrt{2}, 4,4 \sqrt{2}, 6\}$. For constellation $\mathcal{X}$, there are four points uniformly distributed on each circle. The angles of the four points on the same circle are $\{0, \pi / 2, \pi, 3 \pi / 2\}$ or $\{\pi / 4,3 \pi / 4,5 \pi / 4,7 \pi / 4\}$. The optimal value of $\theta$ is found to maximize the distance between $\left(x_{1}, y_{1}\right)$ and the distance between $\left(x_{2}, y_{1}\right)$ simultaneously. It is found that for 4QAM, $\theta=\pi / 12$ and the minimum value of $b$ is maximized to be $(8-8 \cos (\pi / 6))^{2}$.

The conclusion that $\theta=\pi / 12$ is optimal is briefly proved as follows.

1) Consider a pair of points $(x, y)$, where $x \in \mathcal{X}, y \in \mathcal{Y}$, on the same circle. It is straightforward that after constellation rotation by $2 \theta=\pi / 6$, the distance between any point in $\mathcal{X}$ and any point in $\mathcal{Y}$ is greater than or equal to the distance between $\left(x_{1}, y_{1}\right)$.

2) Consider a pair of points $(x, y)$ on the different circles. First, let us consider the two inner circles only. It is easily found from Fig. 1 that $2 \theta=\pi / 6$ is optimal, and distances $\operatorname{dist}_{\left(x_{1}, y_{1}\right)}=\operatorname{dist}_{\left(x_{2}, y_{1}\right)}=\sqrt{8-8 \cos (\pi / 6)}=$ 1.0353. It is also easy to find that the distances between the middle circle and its adjacent circles are greater than 1.0353. Finally, we need to check point pair $(x, y)$ on the two outer circles, for which it is determined that the distance between $(x, y)$ on the two outer circles is always greater than 1.0353. Therefore, $2 \theta=\pi / 6$ is the optimal rotation angle $(\theta=\pi / 12)$ for 4QAM.

Because of the symmetry of $\mathcal{X}$, the minimum value of $b$ is a periodic function of $\theta$ with a period of $\pi / 4$ for QAM and a period of $\pi / Q$ for PSK, with a constellation size $Q$ when $Q$
TABLE I

OPTIMAL RotATION ANGLES FOR SOME COMMONLY USED CONSTELLATIONS

\begin{tabular}{|c|c|}
\hline Constellation & $\theta$ (radians, within one period) \\
\hline 4QAM (QPSK) & $\pi / 12, \quad \pi / 6$ \\
\hline 8PSK & $0.165, \quad 0.227$ \\
\hline 16QAM & $0.322, \quad 0.464$ \\
\hline 16PSK & $0.0825, \quad 0.1138$ \\
\hline 64QAM & $0.322, \quad 0.464$ \\
\hline
\end{tabular}

is an integer power of two. There are two optimal rotation angles for any modulation within one period (e.g., $\pi / 6$ is another optimal rotation angle for 4QAM). For higher order modulation schemes, analysis via an analytical approach is difficult. Through exhaustive numerical search, we determined the optimal rotation angles in the sense of maximizing coding gain for some commonly used constellations and listed them in Table I. Unlike $\boldsymbol{G}_{4}$ given in [8] and [9], for which all QAM constellations have the same optimal rotation angles, the optimal rotation angles of 4QAM and 16QAM are different for $\boldsymbol{G}_{8}$.

\section{Simulation Results}

Error performance of the codes derived using the proposed method is simulated and compared with the theoretical lower bound. The theoretical lower bound is obtained based on a model with one transmit antenna and $N M$ receive antennas that provides full receive diversity. To make it a fair comparison, the average transmission power for this model is normalized to $1 / N$, the same as the STBC system with $N$ transmit antennas. The decision variable for any symbol is expressed as

$$
\hat{s}=\sum_{i=1}^{N M}\left(\sqrt{\frac{\rho}{N}}\left|h_{i}\right|^{2} s+h_{i}^{*} \eta_{i}\right)
$$

where $\eta_{i}, i=1, \ldots, N M$, are samples of independent zeromean complex Gaussian RVs with variance $1 / 2$ per real dimension, $h_{i}$ is the fading coefficient for the $i$ th receive antenna, and $s$ is a symbol. Rate-one ISI-free codes (i.e., orthogonal codes) such as the Alamouti code achieves this bound.

Figs. 2 and 3 show, respectively, the simulated bit-error rate (BER) versus SNR curves for 4QAM and 16QAM systems with five transmit antennas and one receive antenna. Code matrix $\boldsymbol{G}_{5}$ is taken as the first five columns of $\boldsymbol{G}_{8}$. The codes obtained using the proposed method with the optimal constellation-rotation angles were found to have the same BER-SNR slope as the theoretical BER lower bound. This verifies that the codes derived in this letter achieve full diversity. Without rotation, these codes suffer from performance degradation at the high SNR region because of a loss in diversity. Performance of the code proposed in [13] is also shown in Fig. 2. Its code matrix is given as

$$
\left[\begin{array}{ccccc}
s_{1} & e^{j \omega} s_{5} & e^{j \omega} s_{4} & e^{j \omega} s_{3} & e^{j \omega} s_{2} \\
s_{2} & s_{1} & e^{j \omega} s_{5} & e^{j \omega} s_{4} & e^{j \omega} s_{3} \\
s_{3} & s_{2} & s_{1} & e^{j \omega} s_{5} & e^{j \omega} s_{4} \\
s_{4} & s_{3} & s_{2} & s_{1} & e^{j \omega} s_{5} \\
s_{5} & s_{4} & s_{3} & s_{2} & s_{1}
\end{array}\right]
$$

Through computer search, the optimal value of $\omega$, in the sense of maximizing coding gain, is found to be 5.38 radians for 4QAM. 


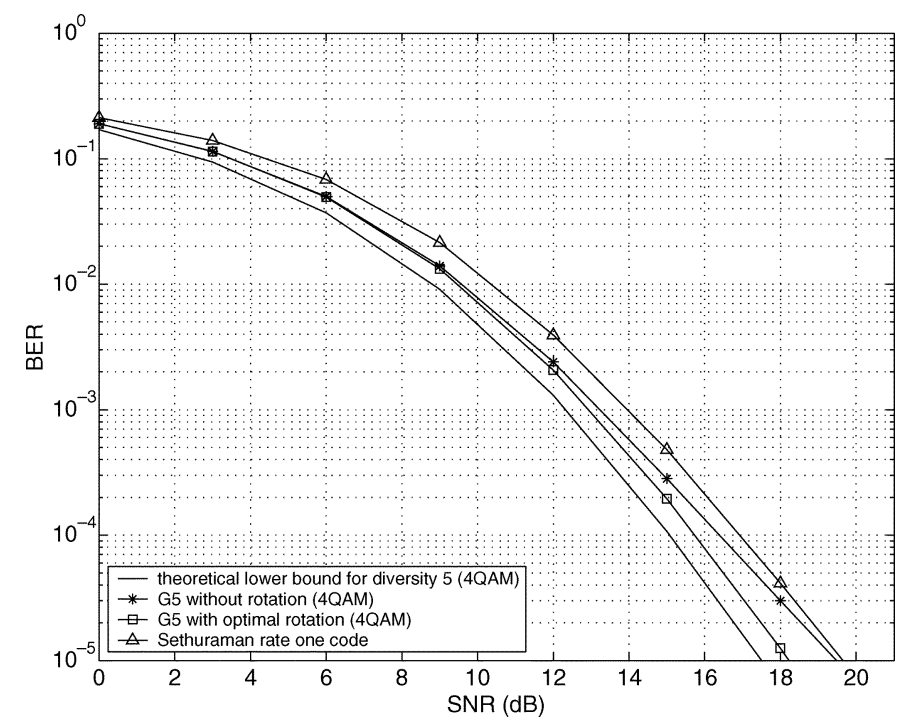

Fig. 2. Performance comparison between $\boldsymbol{G}_{5}$ and theoretical lower bound $(N=5, M=1,2 \mathrm{~b} / \mathrm{s} / \mathrm{Hz})$.

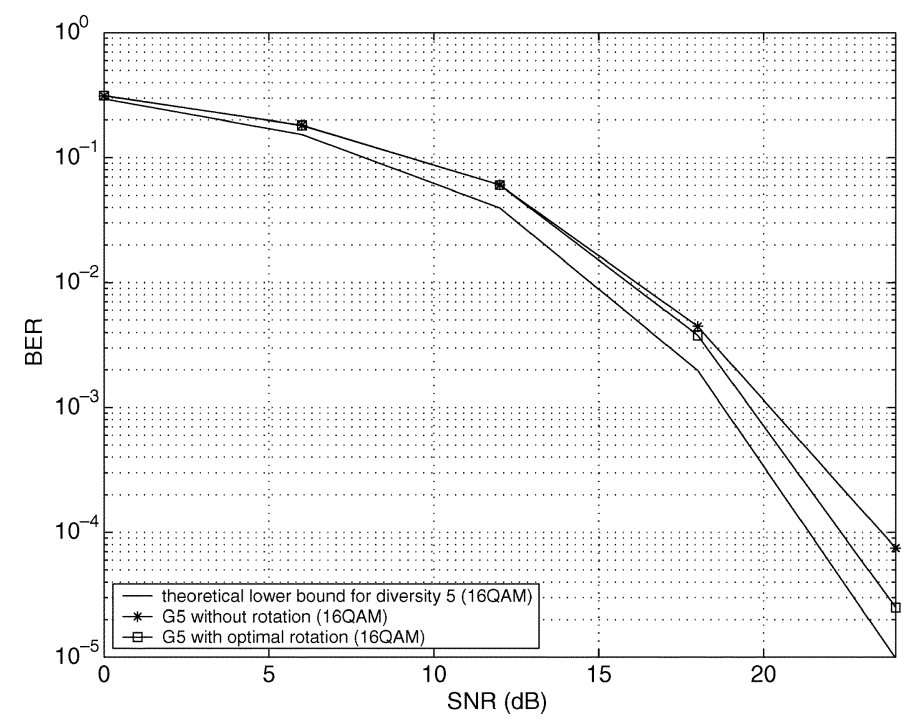

Fig. 3. Performance comparison between $\boldsymbol{G}_{5}$ and theoretical lower bound $(N=5, M=1,4 \mathrm{~b} / \mathrm{s} / \mathrm{Hz})$.

Compared with quasi-orthogonal codes, this code suffers from performance loss due to higher ISI.

\section{CONCLUSION}

We have provided a systematic design method of rate-one STBCs with full diversity for systems with an arbitrary number of transmit antennas. Our code structures are based on the real orthogonal codes, and can be applied to real, as well as complex, signals. Full diversity is achieved by constellation rotation. A decoding method has been provided to lower the decoding complexity of these codes when applied to a MIMO system. Simulation results verified these properties of the codes derived.

\section{REFERENCES}

[1] S. M. Alamouti, "A simple transmit diversity technique for wireless communications," IEEE J. Sel. Areas Commun., vol. 16, no. 10, pp. 1451-1458, Oct. 1998.

[2] V. Tarokh, H. Jafarkhani, and A. R. Calderbank, "Space-time block codes from orthogonal designs," IEEE Trans. Inf. Theory, vol. 45, no. 7, pp. 1456-1467, Jul. 1999.

[3] X.-B. Liang and X.-G. Xia, "On the nonexistence of rate-one generalized complex orthogonal designs," IEEE Trans. Inf. Theory, vol. 49, no. 11, pp. 2984-2989, Nov. 2003.

[4] A. Boariu and D. M. Ionescu, "A class of nonorthogonal rate-one spacetime block codes with controlled interference," IEEE Trans. Wireless Commun., vol. 2, no. 2, pp. 270-276, Mar. 2003.

[5] H. Jafarkhani, "A quasi-orthogonal space-time block code," IEEE Trans. Commun., vol. 49, no. 1, pp. 1-4, Jan. 2001.

[6] O. Tirkkonen, A. Boariu, and A. Hottinen, "Minimal nonorthogonality rate 1 space-time block code for $3+\mathrm{tx}$ antennas," in Proc. IEEE ISSSTA, Sep. 2000, pp. 429-432.

[7] J. Hou, M. H. Lee, and J. Y. Park, "Matrices analysis of quasi-orthogonal space-time block codes," IEEE Commun. Lett., vol. 7, no. 8, pp. 385-387, Aug. 2003.

[8] N. Sharma and C. B. Papadias, "Improved quasi-orthogonal codes through constellation rotation," IEEE Trans. Commun., vol. 51, no. 3 , pp. 332-335, Mar. 2003.

[9] W. Su and X. Xia, "Signal constellations for quasi-orthogonal spacetime block codes with full diversity," IEEE Trans. Inf. Theory, vol. 50, no. 10, pp. 2331-2347, Oct. 2004.

[10] M.Z. A. Khan and B. S. Rajan, "Space-time block codes from coordinate interleaved orthogonal designs," in Proc. IEEE Int. Symp. Inf. Theory, Jul. 2002, p. 275.

[11] M. Z. A. Khan, B. S. Rajan, and M. H. Lee, "On single-symbol and double-symbol decodable STBCs," in Proc. IEEE Int. Symp. Inf. Theory, Jul. 2003, p. 127.

[12] - "Rectangular coordinate interleaved orthogonal designs," in Proc. IEEE Globecom, Dec. 2003, pp. 2004-2009.

[13] B. A. Sethuraman, B. S. Rajan, and V. Shashidhar, "Full-diversity, highrate space-time block codes from division algebras," IEEE Trans. Inf. Theory, vol. 49, no. 10, pp. 2596-2616, Oct. 2003.

[14] O. Damen, A. Chkeif, and J. Belfiore, "Lattice code decoder for spacetime codes," IEEE Commun. Lett., vol. 4, no. 5, pp. 161-163, May 2000.

[15] V. Tarokh, H. Jafarkhani, and A. R. Calderbank, "Space-time codes for high data rate wireless communication: Performance criterion and code construction," IEEE Trans. Inf. Theory, vol. 44, pp. 744-765, Mar. 1998.

[16] L. Xian and H. Liu, "Optimal rotation angles for quasi-orthogonal spacetime codes with PSK modulation," IEEE Commun. Lett., vol. 9, no. 8, pp. 676-678, Aug. 2005. 